\title{
QCD Physics with the CMS Experiment
}

\author{
S. $\operatorname{Cerci}^{1, a, b}$
}

${ }^{1}$ Adiyaman University, Faculty of Arts and Sciences, Department of Physics, 02040 Adiyaman, Turkey.

\begin{abstract}
Jets which are the signatures of quarks and gluons in the detector can be described by Quantum Chromodynamics (QCD) in terms of parton-parton scattering. Jets are abundantly produced at the LHC's high energy scales. Measurements of inclusive jets, dijets and multijets can be used to test perturbative QCD predictions and to constrain parton distribution functions (PDF), as well as to measure the strong coupling constant $\alpha_{S}$. The measurements use the samples of proton-proton collisions collected with the CMS detector at the LHC at various center-of-mass energies of 7, 8 and $13 \mathrm{TeV}$.
\end{abstract}

\section{Introduction}

Quantum chromodynamics (QCD) is the theory of strong interaction describing the interactions between quarks and gluons, so called partons. The QCD physics can be classified into two as "soft QCD" and "hard QCD" depending on the scale of the parton-parton interaction. The soft QCD takes into account the multiple partonic interaction (MPI), underlying event (UE) and fragmentation whereas the hard QCD takes into account the perturbative QCD theory, parton density functions (PDFs), parton shower modeling and initial and final state radiation (ISR and FSR). Many measurements in recent years have been performed for understanding the QCD both in experimental and theoretical aspects. In the theory side, particularly perturbative calculations at next-to-leading order (NLO) are available for most of the observables and Monte Carlo event generators are tuned more precisely with the measurements. In terms of the experimental side, many measurements have been performed with the data taken with the hadron colliders, which can be used to probe the theory in a large phase space.

\section{Soft QCD measurements}

In a high-energy hadron-hadron collision the underlying event (UE) is the activity that is not part of the parton-parton scattering in the collision. Understanding of the UE at high energies accurately is important to have precise measurements of standard model processes and searches for new physics. The underlying event activity is measured with the pp collisions at the centre-of-mass energy of $\sqrt{s}=13 \mathrm{TeV}$ by the CMS Collaboration [1]. The leading charged-particles as well as leading charged-particle jets are used as reference objects in the pseudorapidity region $(|\eta|<2)$. The direction of the leading charged-particle / charged-particle jets is taken into account for defining two transverse regions in $\eta-\phi$ space. In order to construct four observables (transMAX, transMIN, transDIF and

\footnotetext{
${ }^{\mathrm{a}}$ e-mail: Salim.Cerci@ cern.ch

${ }^{\mathrm{b}}$ On behalf of the CMS Collaboration
} 
transAVE) that are useful for describing UE activity the two transverse regions are utilized. The four observables are descibed as following: transMAX (transMIN) is the density in the transverse side with higher (lower) particle density. The difference between transMAX and transMIN densitites is called as transDIF whereas the average of transMAX and transMIN regions is named as transAVE. Figure 1 shows the comparisons of leading jet transverse momentum $p_{T}$ data taken at different centre-of mass energies $\sqrt{s}=0.9,2.76,7$, and $13 \mathrm{TeV}$ with PYTHIA8 (version 8.153) CUETP8M1 [2], CUETP8s1 [2] and Monash [3] tunes and HeRwig++ (version 2.7.0) [4] CUETHs 1 tune. A strong rise in the UE activity with the increasing centre-of-mass energy is observed as predicted by the MC model predictions.
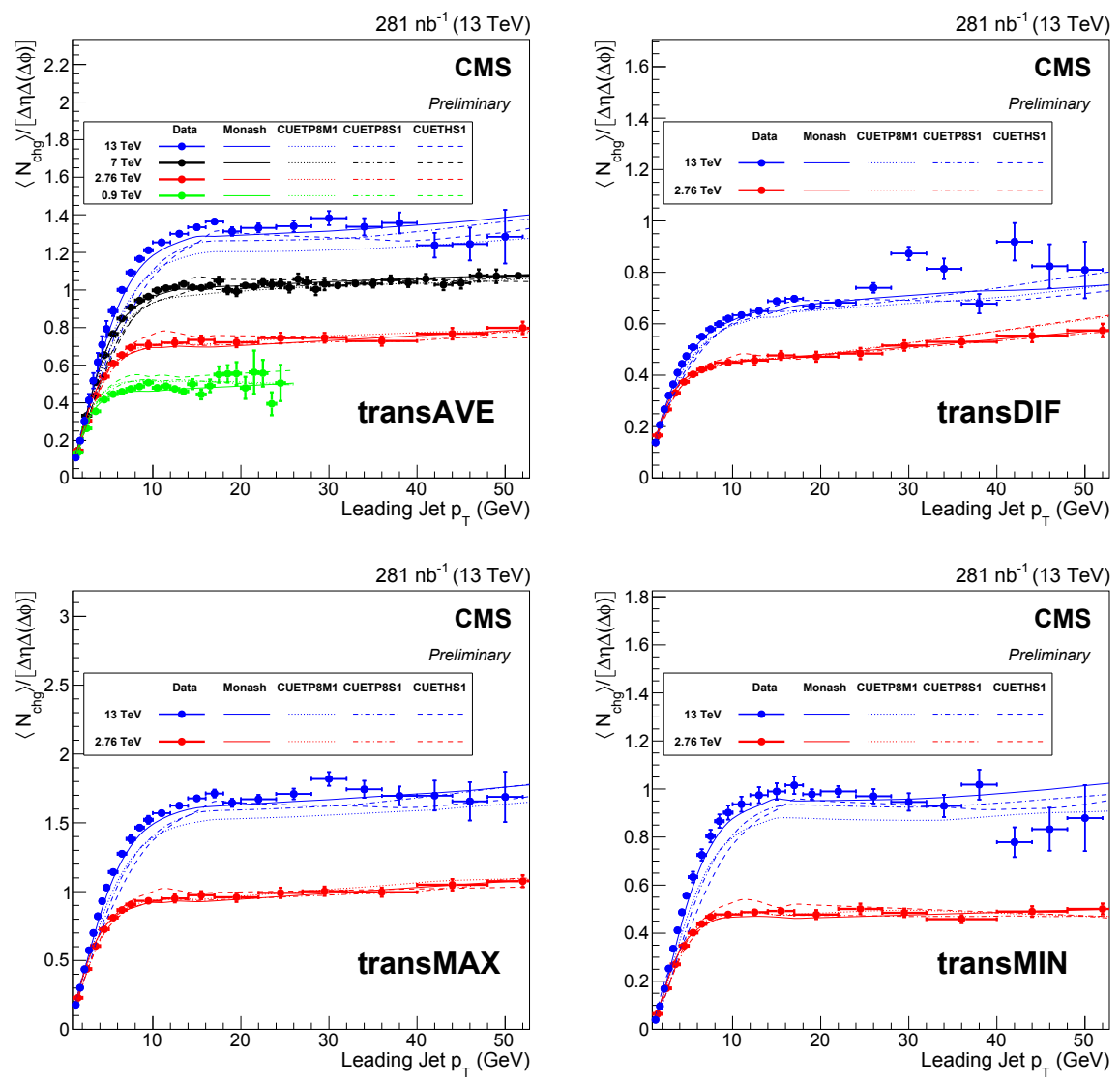

Figure 1. Comparisons between data at different centre-of-mass energies and various MC simulated samples for transAVE, transDIF, transMAX, and transMIN densities.

MPI, the parton saturation scale and the transition between soft and hard QCD may affect the particle and jet transverse momentum spectra at low $p_{T}$ where the fixed-order partonic cross section in theory diverges. The CMS experiment has measured the leading charged particles and leading charged-particle jets with the low transverse momenta in $p p$ collisions at $\sqrt{s}=8 \mathrm{TeV}$ [5].

Azimuthal angle decorrelation between the hadronic jets, which are the visible manifestations of the energetic partons emerging from the underlying processes, is a sensitive probe for better understanding of the QCD radiation in hard processes in high energy particle collisions. Such a measure- 
ment may allow to test various models of parton showers or analytic pQCD resummation methods. According to the QCD prediction the production of two partons has a back-to-back topology in azimuthal plane. Hence, the two jets show a strong correlation in their azimuthal angle. When the higher-order processes are taken into account, the decorrelation, deviation from the back-to-back topology, occurs and more partons manifest in the final state. In the BFKL [6-8] framework such a final state is described with hard parton radiation ordered in rapidity and with no ordering on $p_{T}$. In contrast to DGLAP [9-13], BFKL predicts a strong growth of the inclusive dijet cross section with increasing rapidity interval between the low- $p_{T}$ jets. The measurement of decorrelation in the azimuthal angle between the most forward and the most backward jets (Mueller-Navelet jets) is perfomed by CMS in $p p$ collisions at $\sqrt{s}=7 \mathrm{TeV}$ [14]. The jets are required to have transverse momenta $p_{T}>35 \mathrm{GeV}$ and rapidity $|y|<4.7$, allowing a rapidity separation betwen the MN jets of up to $\Delta y=9.4$. The anti- $k_{t}[15]$ algorithm with a distance parameter $R=0.5$ is used for the reconstruction of jets. Decorrelations are measured as normalised differential cross-sections in $\Delta \phi$ for three rapidity separation in $\Delta y<9.4$, the average cosines of $(\pi-\Delta \phi), 2(\pi-\Delta \phi)$, and $3(\pi-\Delta \phi)$, and ratios of these cosines $\left(C_{2} / C_{1}\right.$ and $\left.C_{3} / C_{2}\right)$. Here $\Delta \phi=\Delta \phi_{1}-\Delta \phi_{2}$ represents the difference between the azimuthal angles $\phi_{1}$ and $\phi_{2}$ of the MN jets. Figure 2 shows comparison of the average cosines as a function of $\triangle y$ with the LL DGLAP-based MC generator SHERPA, NLL BFKL-motivated MC generator HEJ with hadronisation performed with ARIADNE and analytical NLL BFKL calculation.

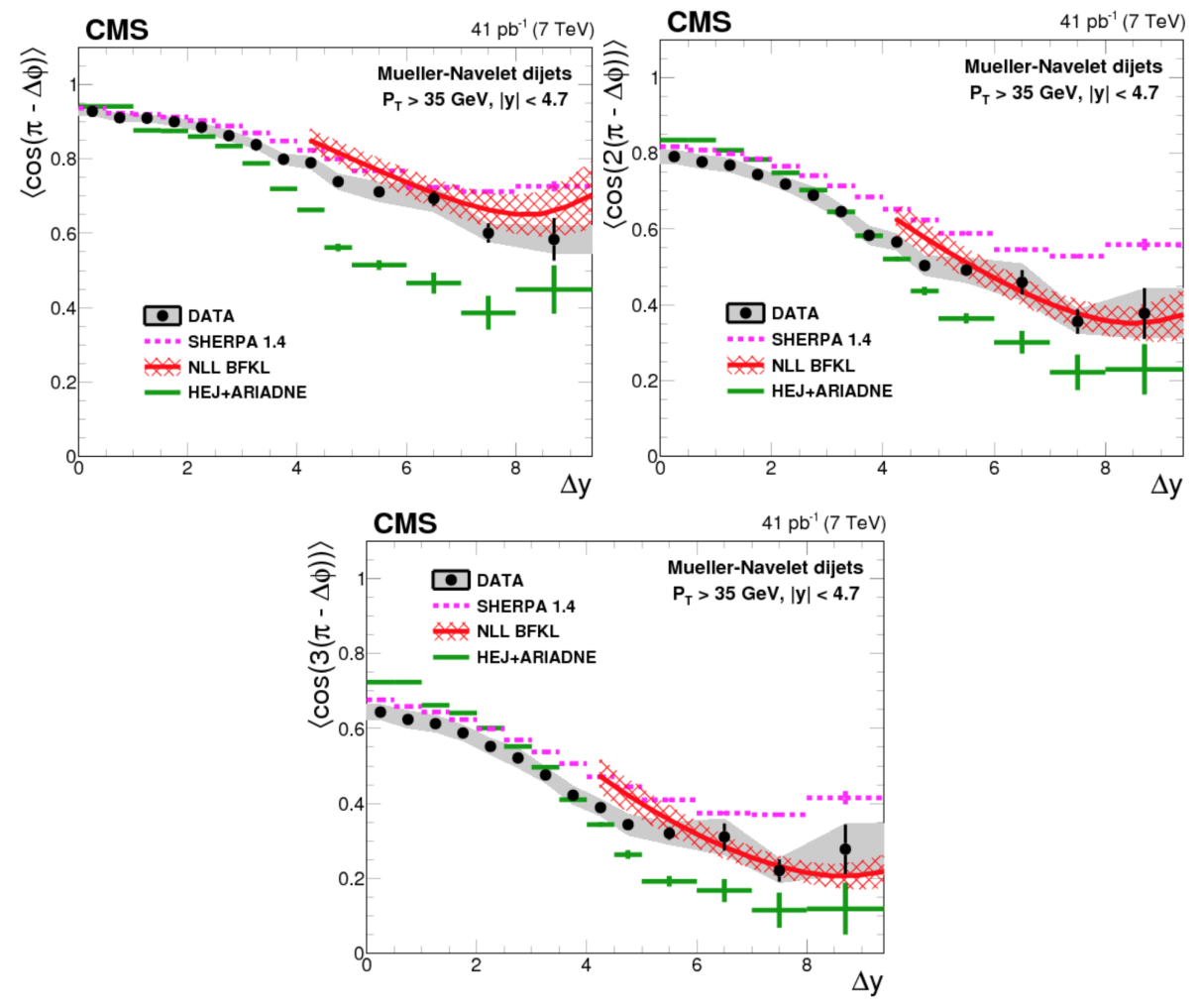

Figure 2. Average cosines as a function of $\Delta y$ compared with the LL DGLAP-based MC generator SHERPA, NLL BFKL-motivated MC generator HEJ with hadronisation performed with ARIADNE and analytical NLL BFKL calculations 
The measured ratios $C_{2} / C_{1}$ and $C_{3} / C_{2}$ as a function of $\Delta y$ are shown in Figure 3. The analytical NLL BFKL calculations gives the best description of data within the experimental and theoretical uncertainties.
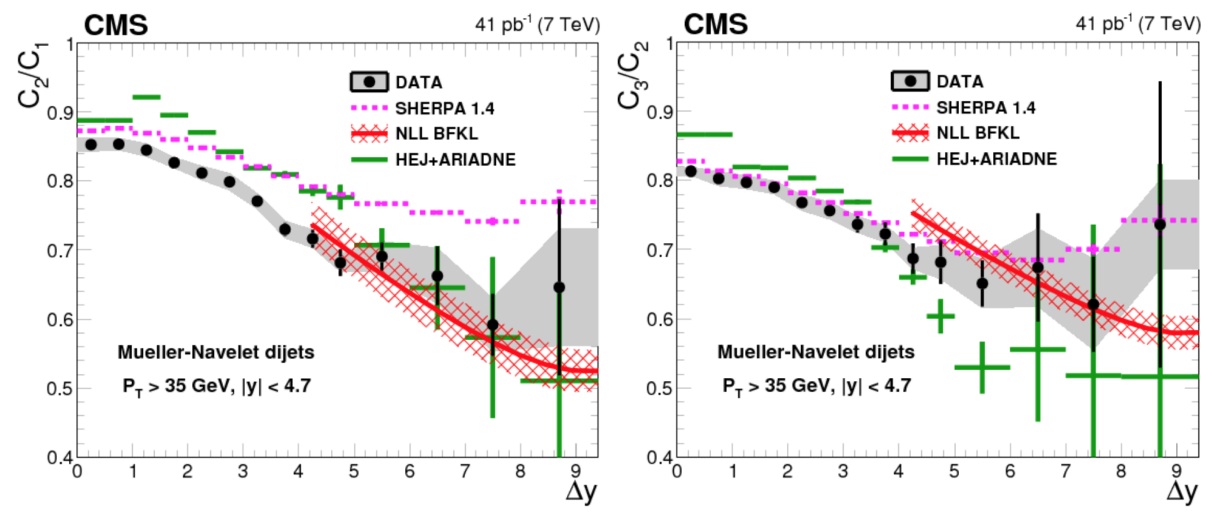

Figure 3. Comparison of measured ratios $C_{2} / C_{1}$ (left) and $C_{3} / C_{2}$ (right) as a function of $\Delta y$ with the LL DGLAPbased MC generator SHERPA, NLL BFKL-motivated MC generator HEJ with hadronisation performed with ARIADNE and analytical NLL BFKL calculations.

\section{Hard QCD measurements}

Most of the measurements performed in LHC extend well into the TeV range, with potential sensitivity to new physics. At the LHC, the principal colliding objects are quarks and gluons and hence QCD becomes the most dominant interaction process with very large cross-section. QCD processes take role as a major background to other standard model processes as well as the new physics searches. Jets are defined as the streams of particles clustered together and observed signatures of quarks and gluons in the detector. Measurements of jet cross section as a function of the jet rapidity $y$ and the jet transverse momentum $\left(p_{T}\right)$ can provide information about all the aspects of the theory and can optimize the hadronization and parton shower models. Such measurements also can be used to constrain the parameters of PDF's at a fixed order of perturbation theory, which for most calculations today is next-to-leading order (NLO) and provide a way of measuring $\alpha_{S}$ at various momentumtransfer scales. The inclusive jet cross-section gives an opportunity to access a wide range of proton momentum fraction, $x$. The large $x$ jet production is dominated by quark jets whereas the lower $x$ jet production is dominated by gluons.

The double-differential inclusive jet cross section as a function of the jet transverse momentum $p_{T}$ and the absolute jet rapidity $|y|$ is measured with the data collected by the CMS detector in $p p$ collisions at $\sqrt{s}=8 \mathrm{TeV}$ [16] and $\sqrt{s}=13 \mathrm{TeV}$ [17]. The $\sqrt{s}=8 \mathrm{TeV}$ measurement is carried out using the anti- $k_{t}$ jet clustering algorithm with distance parameter $R=0.7$ in the range of $|y|<4.7$ for jet transverse momenta $21<p_{T}<2000 \mathrm{GeV}$. The corrected inclusive jet cross section is compared with the predictions from $\mathrm{pQCD}$ at NLO using various sets of PDFs.

Figure 4 shows the measured double-differential cross sections for inclusive jet production as a function of jet $p_{T}$ in the various $|y|$ ranges. Open points represent the data of low- $p_{T}$ analysis, filled points are for the high- $p_{T}$ one. CT10 PDF set is corrected with the non-perturbative (NP) factor for the low- $p_{T}$ data (solid line) and is corrected with the NP and electroweak correction factors for the 


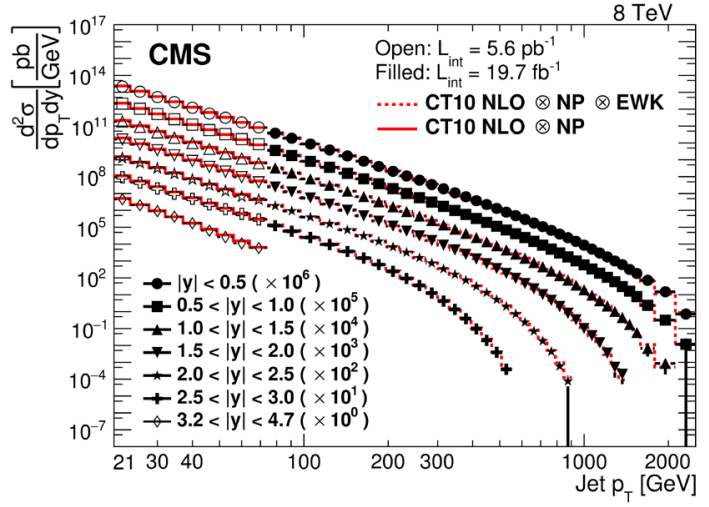

Figure 4. Double-differential inclusive jet cross section as a function of jet $p_{T}$ at $\sqrt{s}=8 \mathrm{TeV}$.

high- $p_{T}$ data (dashed line). Electroweak correction factors are found to be negligible for low- $p_{T}$. Figure 5 shows the ratios of data and alternative predictions to the theory prediction that uses the CT10 PDF set. For comparison, predictions employing five other PDF sets are shown in addition to the total theoretical and total experimental systematic uncertainties. The error bars represent the statistical uncertainty of the data.
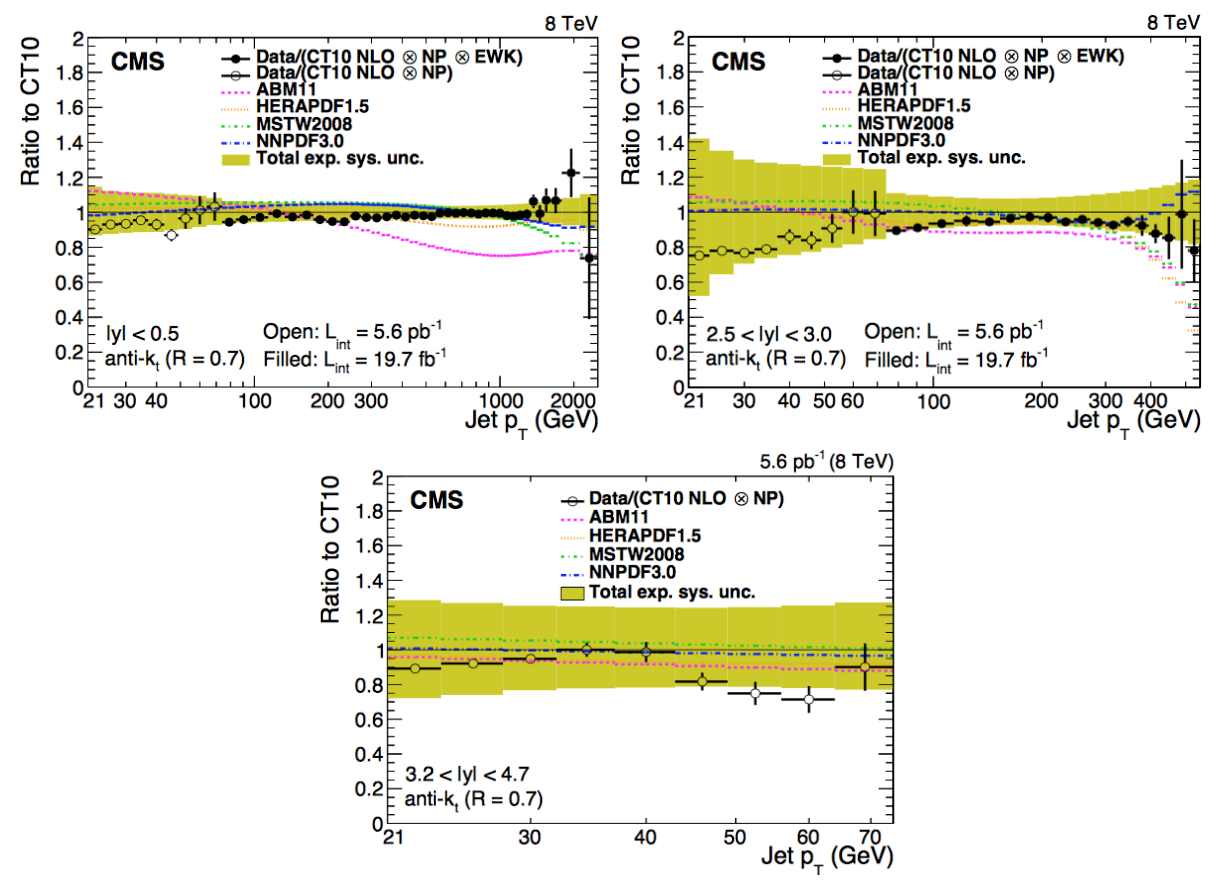

Figure 5. Comparisons of the ratios of data and various predictions to the theory prediction using the CT10 PDF set: for $|y|<0.5$ (top-left), $2.5<|y|<3.0$ (top-right) and $3.2<|y|<4.7$ (bottom). 
The CMS experiment has recently measured the double-differential cross sections at $\sqrt{s}=13 \mathrm{TeV}$ [17]. The anti- $k_{t}$ jet clustering algorithm is used for jet reconstruction for two distance parameters, $R=0.4$ and 0.7 , in a phase space region covering $114 \mathrm{GeV}<p_{T}<2 \mathrm{TeV}$ and $|y|<4.7$. Double-differential inclusive jet cross section as function of jet $p_{T}$ is shown in Figure 6 . The results are compared to the NLO++ predictions using CT14 PDF set, and this theory prediction is corrected with the NP and electroweak effects. Ratio of measured values to theoretical prediction from NLOJet++ using the CT14 PDF set is shown in Figure 7. Predictions employing three other PDF sets are also shown for comparison. The data are found to be consistent with the predictions within the range of $114<p_{T}<2000 \mathrm{GeV}$.

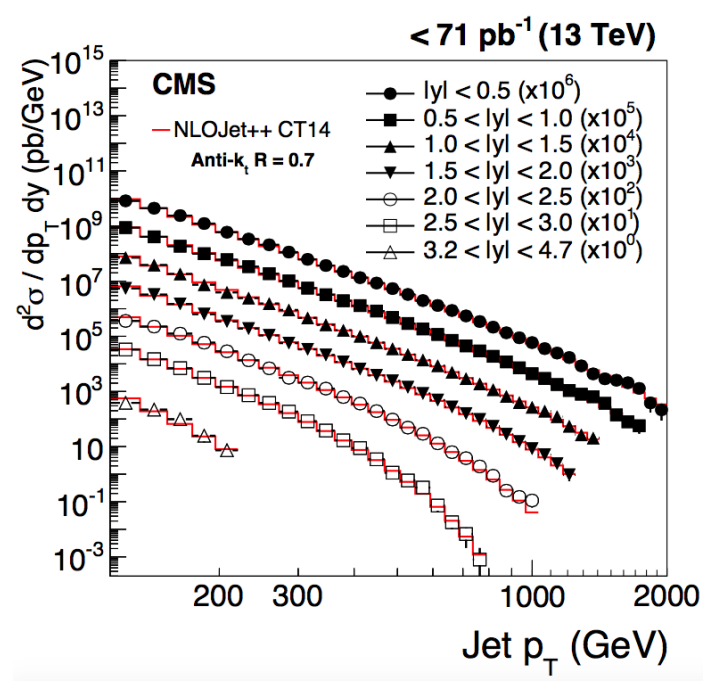

Figure 6. Double-differential inclusive jet cross section as a function of jet $p_{T}$ at $\sqrt{=}=13 \mathrm{TeV}$.

The strong coupling constant $\alpha_{S}$ and PDFs are a key ingredient for precision measurements at hadron colliders. Determination of $\alpha_{s}\left(\mathrm{M}_{\mathrm{Z}}\right)$ is based on the measurement of the inclusive jet production cross section as function of jet $p_{T}$ and rapidity. Such a measurement is performed by CMS as it is shown in Figure 8. The present measurement is in very good agreement with results obtained by previous experiments. The present analysis constrains the $\alpha_{S}(Q)$ running for $Q$ between $86 \mathrm{GeV}$ and $1.5 \mathrm{TeV}$, which is the highest scale at which $\alpha_{S}$ has been measured, to date.

HERA DIS cross section data with the CMS inclusive jet cross section data at $\sqrt{s}=8 \mathrm{TeV}$, provide important constraints on the gluon and valence-quark distributions in the kinematic range studied. These constraints are shown in Figure 9, where the distributions of the gluon and valence quarks are shown at the scales of $Q^{2}=1.9 \mathrm{GeV}^{2}$. Gluon PDF uncertainty is reduced in the wide range of $x$, while for quark PDF uncertainty improvement is demonstrated for large $x$.

\section{Summary}

CMS has been delivering a wealth of precise standard model results since the start of the LHC Run 2 era. The soft and hard QCD results presented here provide a deep insight to understand both perturbative and non-perturbative QCD physics in LHC as it reaches higher energy. Results can also be utilized in improving MC models, determining strong coupling constant and constraining PDFs. 

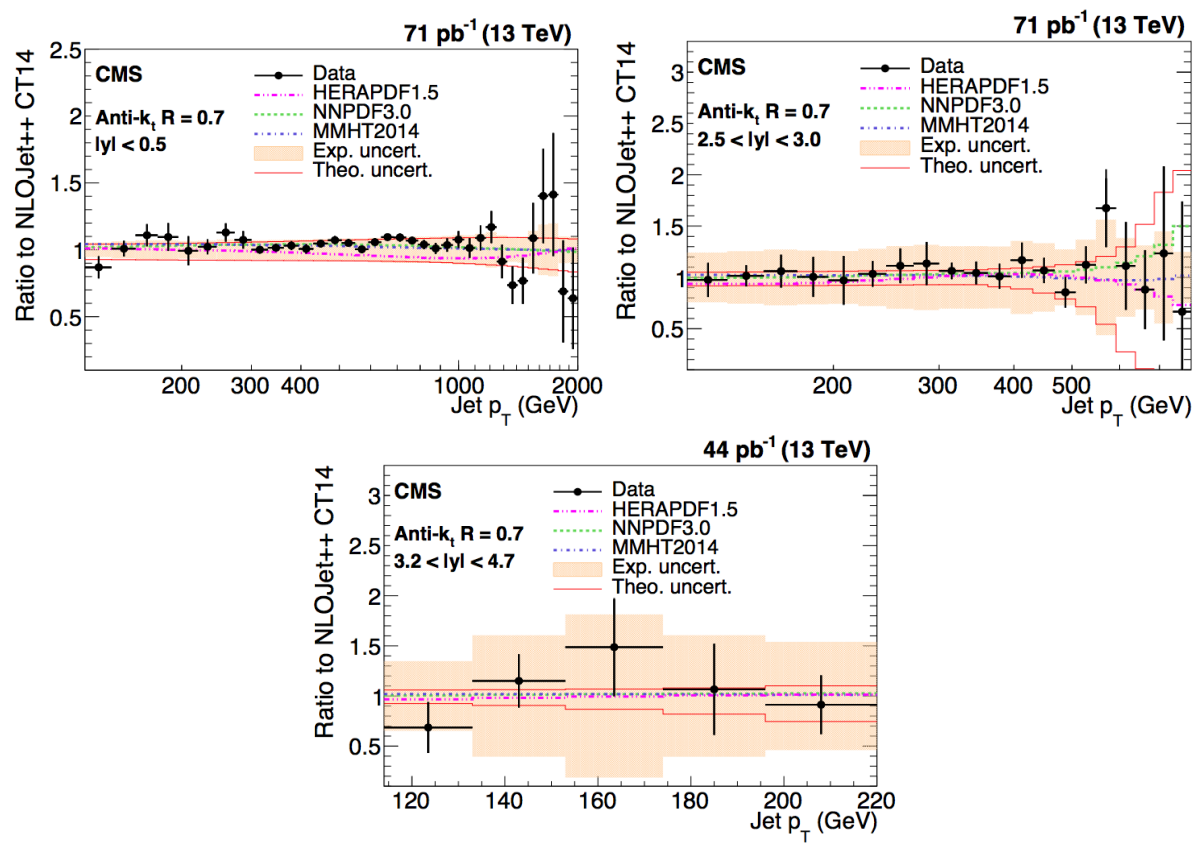

Figure 7. Comparisons of the ratios of data and various predictions to the theory prediction using the CT14 PDF set: for $|y|<0.5$ (top-left), $2.5<|y|<3.0$ (top-right) and $3.2<|y|<4.7$ (bottom).

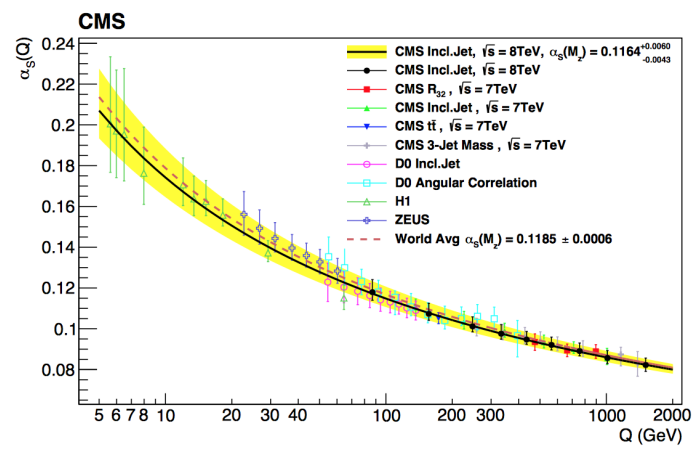

Figure 8. Running of the strong coupling constant $\alpha_{S}$ as measured in hadron colliders: Tevatron (D0), HERA (H1 and ZEUS) and LHC (CMS).

The CMS collaboration has already provided extensive list of measurements on the jets as well as the strong coupling constant based on different observables. All measurements are found to have good agreement with the world average value and the running of $\alpha_{S}$ is confirmed at the TeV scale. The inclusive jet spectrum is used to provide additional constraints to PDFs, thus improving the gluon and the valence-quark distributions. A significant reduction of PDF uncertainties has been observed. 

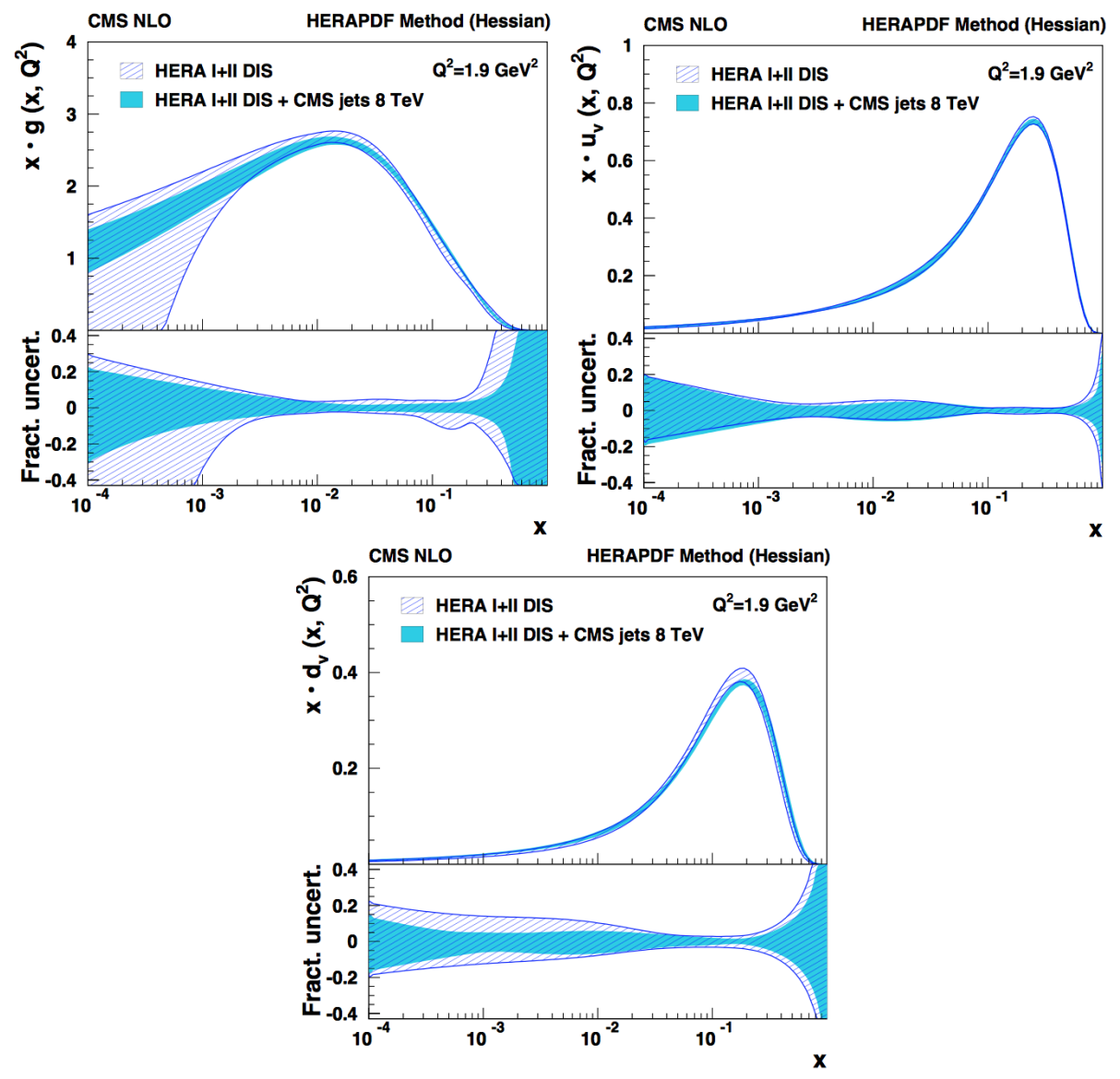

Figure 9. Distributions of gluon (top-left), u-valence quark (top-right), and d-valence quark (bottom) as functions of $x$ at $Q^{2}=1.9 \mathrm{GeV}^{2}$.

\section{Acknowledgments}

The author would like to acknowledge the Adiyaman University Scientific Activity Foundation and the organizers of ICNFP2016 for the funding. 


\section{References}

[1] CMS Collaboration, CMS Physics Analysis Summary CMS PAS FSQ-15-007, (2016)

[2] V. Khachatryan et al. [CMS Collaboration], Eur. Phys. J. C 76, no. 3, 155, (2016)

[3] P. Skands, S. Carrazza, and J. Rojo, EPJ C 74, no. 8 (2014)

[4] M. Bahr et al., Eur. Phys. J. C 58, 639 (2008)

[5] V. Khachatryan et al. [CMS Collaboration], Phys. Rev. D 92, 112001 (2015)

[6] E.A. Kuraev, L.N. Lipatov and V.S. Fadin, Sov. Phys. JETP 44 443, (1976)

[7] E.A. Kuraev, L.N. Lipatov and V.S. Fadin, Sov. Phys. JETP 45 199, (1977)

[8] I.I. Balitsky and L.N. Lipatov, Sov. J. Nucl. Phys. 28 822, (1978)

[9] V.N. Gribov and L.N. Lipatov, Sov. J. Nucl. Phys. 15 438, (1972)

[10] V.N. Gribov and L.N. Lipatov, Sov. J. Nucl. Phys. 15 675, (1972)

[11] L.N. Lipatov, Sov. J. Nucl. Phys. 20 94, (1975)

[12] G. Altarelli and G. Parisi, Nucl. Phys. B 126 298, (1977)

[13] Y.L. Dokshitzer, Sov. Phys. JETP 46 641, (1977)

[14] V. Khachatryan et al. [CMS Collaboration], JHEP 08 139, (2016)

[15] M. Cacciari, G.P. Salam and G. Soyez, JHEP 04 063, (2008)

[16] V. Khachatryan et al. [CMS Collaboration], Submitted to JHEP, arXiv:1609.05331 [hep-ex], (2016)

[17] V. Khachatryan et al. [CMS Collaboration], Eur. Phys. J. C 76 451, (2016) 